\title{
Cross Pins versus Endobutton Femoral Fixation in Hamstring Anterior Cruciate Ligament Reconstruction: Minimum 4-Year Follow-Up
}

\author{
Chae-Gwan Kong, $\mathrm{MD}^{1}$, Yong In, $\mathrm{MD}^{2}$, Geon-Hyeong Kim, $\mathrm{MD}^{1}$ and Chi-Young Ahn, $\mathrm{MD}^{1}$ \\ Department of Orthopaedic Surgery, ${ }^{1}$ Uijongbu St. Mary’s Hospital, ${ }^{2}$ Seoul St. Mary’s Hospital, The Catholic University of Korea, Seoul, Korea
}

\begin{abstract}
Purpose: We aimed to compare cross-pin fixation and Endobutton femoral fixation for hamstring anterior cruciate ligament (ACL) reconstruction with respect to clinical and radiographic results, including tunnel widening and the progression of knee osteoarthritis (OA).

Materials and Methods: Between August 2002 and August 2005, 126 autogenous hamstring ACL reconstructions were performed using either cross pins or Endobutton for femoral fixation. Fifty-six of 75 patients in the cross-pin group and 35 of 51 patients in the Endobutton group were followed up for a minimum of 4 years. We compared the clinical and radiological results between the groups using the International Knee Documentation Committee (IKDC) evaluation form, the KT-2000 arthrometer side to side difference, the amount of tunnel widening and the advancement of OA on radiographs.

Results: There were no significant differences in the IKDC grades between the groups at the 4 year follow-up. There was no significant difference in the side to side difference according to KT-2000 arthrometer testing. Also, there were no significant differences in terms of tunnel widening or advancement of OA on radiographs.

Conclusions: Endobutton femoral fixation showed good results that were comparable to those of cross pins fixation in hamstring ACL reconstruction.
\end{abstract}

Key words: Anterior cruciate ligament reconstruction, Hamstring tendon, Graft fixation, Tunnel widening.

\section{Introduction}

The selection of a graft and the method of graft fixation are critical in anterior cruciate ligament (ACL) reconstruction surgery ${ }^{1,2}$. ACL reconstruction with hamstring tendon provides sufficient strength for early rehabilitation and activity ${ }^{3,4}$. Indeed,

Received April 28, 2011; Revised (1st) June 30, 2011;

(2nd) September 15, 2011; Accepted November 6, 2011.

Correspondence to: Yong In, MD.

Department of Orthopaedic Surgery, Seoul St. Mary's Hospital, The Catholic University of Korea, 222 Banpodae-ro, Seocho-gu, Seoul 137701 , Korea.

Tel: +82-2-2258-2838, Fax: +82-2-535-9834

Email: iy1000@catholic.ac.kr

This is an Open Access article distributed under the terms of the Creative Commons Attribution Non-Commercial License (http://creativecommons.org/licenses/by-nc/3.0/) which permits unrestricted non-commercial use, distribution, and reproduction in any medium, provided the original work is properly cited. harvest of the hamstring tendon does not injure the extensor mechanism and produces less pain than harvest of the patellar tendon. However, the tendon to bone healing and the fixation method can become an issue with the hamstring tendon, which is unlike the patellar tendon.

A large number of femoral tunnel fixation systems have been introduced and used in ACL reconstruction surgery with autogenous hamstring tendon. In general, three types of ACL graft fixation mechanisms for the femoral tunnel exist, and these can be classified according to the amount, application site and distribution: compression, expansion and suspension ${ }^{5}$. Compression fixation is achieved with interference screws. A typical example of expansion devices is the cross pin system and that of a cortical suspension device is the Endobutton CL (Smith \& Nephew Endoscopy, Andover, MA, USA).

The Endobutton system has been widely used as a fixation device for a quadruple hamstring graft ${ }^{6}$. However, there is a certain elasticity in the graft-implant complex, and the fixation point of the Endobutton system is relatively far from the joint line, resulting in graft-tunnel motion of up to $3 \mathrm{~mm}$ under a 
physiologic load ${ }^{7,8)}$. It has been reported that this intra-tunnel motion has been associated with tunnel widening ${ }^{9}$.

Clark et al. ${ }^{10)}$ described transcondylar femoral cross pin fixation. In the case of cross pin fixation, the anchor point is close to the joint, and the pin in the tunnel itself has the effect of graft expansion. For this reason, it has been reported that tunnel widening less commonly occurs with the cross pin system ${ }^{11,12}$. However, a correlation between tunnel widening and knee laxity has not been shown ${ }^{13-16)}$.

The purpose of this retrospective study was to compare the clinical and radiological results of Endobutton femoral fixation for reconstruction with those of cross pin fixation.

\section{Materials and Methods}

After Institutional Review Board approval, we performed a retrospective review of 131 cases of single incision, arthroscopically assisted, primary ACL reconstruction with quadrupled semitendinosus and gracilis tendon autograft from August 2002 to August 2005. Patients with osteoarthritis (OA) at the time of surgery and those with multi-ligaments injury were excluded. One hundred twenty-six autogenous hamstring ACL reconstructions were performed using either cross pins or Endobutton CL for femoral fixation with an $8 \mathrm{~mm}$ diameter tunnel width. The early operations were mainly performed with cross pins and the latter operations were mainly performed with an Endobutton CL. Fifty-six of the 75 cross pin patients and 35 of the 51 Endobutton patients were followed up for a minimum of 4 years. The patients were divided into two groups according to the method of femoral tunnel fixation. The patients in whom we used the Rigidfix femoral cross pin fixation system (Mitek, Norwood, MA, USA) and the Intrafix (Mitek) device for tibial fixation were classified as the Cross pin group. The patients in whom we used the Endobutton CL suspensory femoral fixation and a bioabsorbable interference screw with a metal screw and washer for tibial fixation were classified as the Endobutton group.

Table 1. Comparison of the Demographic Data between the Two Groups

\begin{tabular}{lccc}
\hline & $\begin{array}{c}\text { Cross pins } \\
\text { group }(\mathrm{n}=56)\end{array}$ & $\begin{array}{c}\text { Endobutton } \\
\text { group }(\mathrm{n}=35)\end{array}$ & p-value \\
\hline Age at surgery $(\mathrm{yr})$ & $31.2 \pm 10.9$ & $30.2 \pm 10.0$ & 0.664 \\
Male:Female & $52: 4$ & $30: 5$ & 0.298 \\
Follow-up (mo) & $57.4 \pm 8.4$ & $55.5 \pm 7.4$ & 0.287 \\
Meniscal tears & 22 & 16 & 0.545 \\
\hline
\end{tabular}

Table 1 shows the patient demographics.

Clinical follow-up on 91 knees (Cross pin group, 56; Endobutton group, 35) included the International Knee Documentation Committee (IKDC) evaluation form classification and KT2000 arthrometer measurements. The radiographic evaluation included the anteroposterior (AP) and lateral radiographs. For correction of magnification, we used a $10 \mathrm{~mm}$ metal ball. The ball was taped to the skin on the midline of the lateral side of the knee on the AP radiograph, and the ball was taped posteriorly in the midline of the knee on the lateral radiograph. Three orthopaedic surgeons who were independent of the surgical team digitally measured the femoral and tibial tunnel width at $1 \mathrm{~cm}$ from the aperture of the femoral and tibial tunnels at the joint. The measurement was performed perpendicular to the long axis of the tunnels. The tunnel widening was measured on the immediate postoperative radiographs and the last follow-up radiographs. The average data of three measurements were used for statistical analysis. To compare the advancement of OA, the degree of OA using the Kellgren-Lawrence grade ${ }^{17)}$ was measured on the preoperative and last follow-up radiographs. The patients who developed arthritic changes underwent additional standing AP radiography.

The same surgical procedure, including conditioning the graft and graft preparation with sutures, was applied to both groups. The location of the tibial tunnel was in the posteromedial aspect of the ACL footprint and a trans-tibial femoral tunnel was drilled as far posterolaterally as possible. The starting point of the tibial guide was made at the midpoint of the tibial face between the tibial tubercle and the posteromedial aspect of the tibia. Regarding the femoral tunnel fixation method, the Cross pin group (Rigidfix system) had $30 \mathrm{~mm}$ of the graft within the tunnel and 2 poly-L-lactide, $3.3 \mathrm{~mm}$ bioabsorbable cross pins placed across the graft according to the manufacturer's protocol. In the Endobutton group (Endobutton CL system), a 15-40 $\mathrm{mm}$ closed loop Endobutton was used according to the tunnel length of the femoral cortex. The same rehabilitation protocol was applied in both groups following reconstruction. Full weight bearing ambulation with crutches was allowed immediately after operation except for the meniscal repair patients. Gradual full range of motion with a brace was allowed from the 2 nd postoperative week.

Statistical comparisons were performed between the groups. The normally distributed continuous variables were analyzed using the student's $t$-test. Ordinal variables were analyzed using the chi-square test. The level of statistical significance was set at $\mathrm{p}<0.05$ for all the tests. All the statistical analyses were performed 
with SPSS ver. 13.0 (SPSS, Inc., Chicago, IL, USA).

\section{Results}

Both groups had comparable demographic data (Table 1). All the patients in both groups showed abnormal (C) or severely abnormal (D) grades of the IKDC classification preoperatively. At 4 years follow-up, the percentage of normal (A) and nearly normal (B) patients was not significantly different in both the Cross pin group (46 of 56, 82.1\%) and the Endobutton group $(28$ of $35,80 \%)(p=0.799)$ (Table 2$)$. With respect to the side-to-side difference on the KT-2000 arthrometer testing, no significant differences were found between the Cross pin group $(2.1 \pm 1.0 \mathrm{~mm})$ and the Endobutton group $(2.5 \pm 1.1 \mathrm{~mm})$ $(\mathrm{p}=0.09)$. The percentage of normal (A) and nearly normal (B) patients in instrumented ligament examination item of the IKDC classification were not different in both the Cross pin group (55 of $56,98.2 \%$ ) and the Endobutton group (34 of 35, 97.1\%) ( $\mathrm{p}=1.000$ ) (Table 3). Both groups showed similar improvements in terms of laxity at the last follow-up.

On the tunnel width measurements using radiographs taken at 4 years postoperatively, both the femoral and tibial tunnels were enlarged sagittally and coronally in both groups (Fig. 1). There were no significant differences in the amount of femoral and tibial tunnel widening in both groups (Table 4). At the final follow-up, 5 patients in the Cross pin group and 2 patients in the

Table 2. International Knee Documentation Committee Scores Comparison between the Two Groups

\begin{tabular}{cccccc}
\hline & \multicolumn{2}{c}{ Preoperative } & & \multicolumn{2}{c}{ 4 years postoperative } \\
\cline { 2 - 3 } \cline { 5 - 6 } & $\begin{array}{c}\text { Cross pin } \\
\text { group }(\mathrm{n}=56)\end{array}$ & $\begin{array}{c}\text { Endobutton } \\
\text { group }(\mathrm{n}=35)\end{array}$ & & $\begin{array}{c}\text { Cross pin } \\
\text { group }(\mathrm{n}=56)\end{array}$ & $\begin{array}{c}\text { Endobutton } \\
\text { group }(\mathrm{n}=35)\end{array}$ \\
\hline A & 0 & 0 & & 4 & 3 \\
B & 0 & 0 & & 42 & 25 \\
C & 27 & 14 & & 7 & 4 \\
D & 29 & 17 & & 3 & 3 \\
\hline
\end{tabular}

A: normal, B: nearly normal, C: abnormal, D: severely abnormal.

Table 3. KT-2000 Side to Side Differences at 4 Years Follow-up

\begin{tabular}{ccc}
\hline $\begin{array}{c}\text { Side to side difference } \\
(\mathrm{mm})\end{array}$ & $\begin{array}{c}\text { Cross pins group } \\
(\mathrm{n}=56)\end{array}$ & $\begin{array}{c}\text { Endobutton group } \\
(\mathrm{n}=35)\end{array}$ \\
\hline$<3$ & 46 & 27 \\
$3-5$ & 9 & 7 \\
$>5$ & 1 & 1 \\
$>10$ & 0 & 0 \\
\hline
\end{tabular}

Endobutton group showed radiographic advancement of OA of 1 grade according to the Kellgren-Lawrence scale.

\section{Discussion}

ACL reconstruction surgery is one of the most common procedures in sports traumatology ${ }^{18)}$. The selection of the graft depends on the surgeon's preference and the available tissues ${ }^{19)}$. Among the autogenous tissues, the most commonly used grafts are currently the patellar tendon and the quadrupled hamstrings. Each of these grafts has been shown to have sufficient load-tofailure strength and stiffness to replace the $\mathrm{ACL}^{3)}$. Graft fixation is also important. To promote an early return to daily life and sports activities, a strong graft with fixation that can endure the strain put on the knee during early rehabilitation is essential ${ }^{20)}$.

ACL graft fixation mechanisms can be classified according to the amount, application site and distribution of forces that resist graft pullout. To et al. ${ }^{4)}$ reported in a cadaver study that the stiffness of a graft-graft fixation complex is largely dependent on the fixation method and less on the graft itself, and that the close-to-joint transcondylar fixation method in that respect was
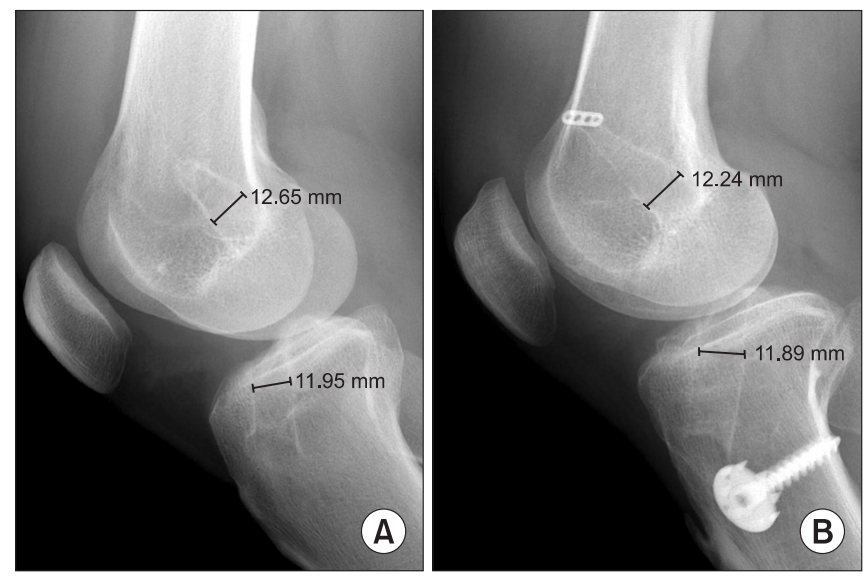

Fig. 1. Examples of a (A) Cross pin group patient's radiographs and an (B) Endobutton group patient's radiographs with digital tunnel measurements.

Table 4. Tunnel Widening in the Femur and Tibia at the Last Follow-up

\begin{tabular}{lccc}
\hline \multicolumn{1}{c}{ View } & $\begin{array}{c}\text { Cross pin } \\
\text { group }(\mathrm{n}=56)\end{array}$ & $\begin{array}{c}\text { Endobutton } \\
\text { group }(\mathrm{n}=35)\end{array}$ & p-value \\
\hline Femoral AP $(\mathrm{mm})$ & $11.3 \pm 0.9$ & $11.3 \pm 1.2$ & 0.848 \\
Femoral lateral $(\mathrm{mm})$ & $11.4 \pm 0.9$ & $11.7 \pm 0.8$ & 0.116 \\
Tibial AP $(\mathrm{mm})$ & $11.5 \pm 0.7$ & $11.5 \pm 0.9$ & 0.986 \\
Tibial lateral $(\mathrm{mm})$ & $12.0 \pm 1.0$ & $11.9 \pm 1.0$ & 0.816 \\
\hline AP: anteroposterior. & & &
\end{tabular}


superior to the Endobutton system. Milano et al..$^{5)}$ used 9 different kinds of femoral fixation methods and showed that the greatest failure loads were reported for Transfix (Arthrex, Naples, FL, USA). Rigidfix and Endobutton were included in an intermediate subset. In another animal experiment, the interference screw and the Rigidfix fixation demonstrated inferior fixation biomechanics compared to the Bio-Transfix (Arthrex) and Endobutton techniques ${ }^{21)}$.

According to previous studies, tunnel widening after ACL reconstruction was more likely to develop with the use of hamstring grafts compared to bone-patellar tendon-bone (BTB) grafts ${ }^{9,14}$. The two main reasons for this are: fixation method using interference screws and bone to bone healing. The interference screw provides fixation close to the aperture of the tunnel and the bony end of the BTB readily integrates with the surrounding bony tunnel. In addition, there was a study that showed that bioabsorbable interference screws can cause more tunnel widening compared to metal screws ${ }^{22)}$.

Several theories have been developed to account for tunnel widening following ACL reconstruction, including mechanical and biological contributions. Within the tunnel, up and down motion (a bungee effect) and side to side motion (the motion of windshield wipers) can occur. Extravasation of synovial fluid that contains various cytokines into the tunnel around the graft may be increased by this motion and this interferes with the soft tissue-to-bone healing ${ }^{23}$. In the suspensory fixation system, these interactions are likely to occur.

Baumfeld et al. ${ }^{11)}$ reported that the 2 cross pin fixation method called Rigidfix showed less femoral tunnel widening compared to the Endobutton method, and tunnel widening could lead to failure after reconstruction surgery. Fauno and Kaalund ${ }^{12)}$ reported that tunnel widening is influenced by the mechanical properties of the implants and more patients with increased knee laxity were in the extracortical fixation (Endobutton fixation) group compared to the close-to-joint fixation (Transfix) group. However, the clinical results were considered successful in both groups.

In the present study, we compared 2 different fixation systems and each had its own characteristics. One is a double cross pins system called Rigidfix, and the other is a suspensory system called Endobutton. According to previous studies, significantly more femoral tunnel widening developed with the use of a suspensory system compared to that of the double cross pin system for quadrupled hamstring grafts ${ }^{11,12)}$. In this current study, we attempted to verify this phenomenon and its relationship with the clinical results obtained in our patients. However, there was no difference in the femoral and tibial tunnel widening between the 2 femoral fixation systems. Additionally, there were no statistical differences in the functional outcomes, such as the IKDC classification and the KT-2000 arthrometer side to side difference. The clinical results were considered successful in both groups.

There are disagreements regarding the timeline of tunnel widening. Some authors reported that tunnel widening is an acute phenomenon that occurs during the first 6 weeks after surgery, while others reported that it continues to develop over the first 1 to 2 years following ACL reconstruction ${ }^{24-27}$. Simonian et al. ${ }^{16)}$ observed that tunnel widening did not develop between the first 3 and 12 months. Fink et al. ${ }^{25)}$ reported that tunnel widening peaked at 6 weeks following graft fixation. Pinczewski et al. ${ }^{27)}$ have observed that tunnel widening progressed up until 2 years after reconstruction. In this study, both the cross pin group and the Endobutton group showed similar tunnel widening at 4 years postoperatively.

The limitations of our study are the followings. This study was a retrospective study, and the follow up loss rate was high (27.8\%). In addition, we did not measure serial tunnel widening during the follow up period of more than 4 years. Identification of the serial change in tunnel widening and its relationship with the clinical results in both groups would be an interesting study with long-term follow-up. In this study, we used two different graft fixation methods for the tibial tunnel for each group. Although there have been disagreements about the relationship between tibial tunnel widening and fixation methods ${ }^{10,18)}$, the difference in the tibial tunnel fixation method could have affected the development of femoral tunnel widening in this study. Intrafix and bioabsorbable screws were located inside the tibial tunnel and each device would have affected the tunnel widening in a different way. Serial follow-up data of postoperative tunnel widening should have been clearly compared with those of the last follow-up.

\section{Conclusion}

Endobutton femoral fixation showed good results comparable to those of cross pin fixation in hamstring ACL reconstruction. Tunnel widening following reconstruction developed in both groups, and this did not lead to failure of surgery. We conclude that both cross pin and Endobutton CL are useful materials for femoral tunnel fixation in hamstring ACL reconstruction surgery. 


\section{References}

1. Brand J Jr, Weiler A, Caborn DN, Brown CH Jr, Johnson DL. Graft fixation in cruciate ligament reconstruction. Am J Sports Med. 2000;28:761-74.

2. Milano G, Mulas PD, Sanna-Passino E, Careddu GM, Ziranu F, Fabbriciani C. Evaluation of bone plug and soft tissue anterior cruciate ligament graft fixation over time using transverse femoral fixation in a sheep model. Arthroscopy. 2005;21:532-9.

3. Hamner DL, Brown CH Jr, Steiner ME, Hecker AT, Hayes WC. Hamstring tendon grafts for reconstruction of the anterior cruciate ligament: biomechanical evaluation of the use of multiple strands and tensioning techniques. J Bone Joint Surg Am. 1999;81:549-57.

4. To JT, Howell SM, Hull ML. Contributions of femoral fixation methods to the stiffness of anterior cruciate ligament replacements at implantation. Arthroscopy. 1999;15:379-87.

5. Milano G, Mulas PD, Ziranu F, Piras S, Manunta A, Fabbriciani C. Comparison between different femoral fixation devices for ACL reconstruction with doubled hamstring tendon graft: a biomechanical analysis. Arthroscopy. 2006;22:660-8.

6. Barrett GR, Papendick L, Miller C. Endobutton button endoscopic fixation technique in anterior cruciate ligament reconstruction. Arthroscopy. 1995;11:340-3.

7. Hoher J, Livesay GA, Ma CB, Withrow JD, Fu FH, Woo SL. Hamstring graft motion in the femoral bone tunnel when using titanium button/polyester tape fixation. Knee Surg Sports Traumatol Arthrosc. 1999;7:215-9.

8. Jorgensen U, Thomsen HS. Behavior of the graft within the bone tunnels following anterior cruciate ligament reconstruction, studied by cinematic magnetic resonance imaging. Knee Surg Sports Traumatol Arthrosc. 2000;8:32-5.

9. L'Insalata JC, Klatt B, Fu FH, Harner CD. Tunnel expansion following anterior cruciate ligament reconstruction: a comparison of hamstring and patellar tendon autografts. Knee Surg Sports Traumatol Arthrosc. 1997;5:234-8.

10. Clark R, Olsen RE, Larson BJ, Goble EM, Farrer RP. Crosspin femoral fixation: a new technique for hamstring anterior cruciate ligament reconstruction of the knee. Arthroscopy. 1998;14:258-67.

11. Baumfeld JA, Diduch DR, Rubino LJ, Hart JA, Miller MD, Barr MS, Hart JM. Tunnel widening following anterior cruciate ligament reconstruction using hamstring autograft: a comparison between double cross-pin and suspensory graft fixation. Knee Surg Sports Traumatol Arthrosc. 2008;16:1108-13.

12. Fauno P, Kaalund S. Tunnel widening after hamstring anterior cruciate ligament reconstruction is influenced by the type of graft fixation used: a prospective randomized study. Arthroscopy. 2005;21:1337-41.

13. Fahey M, Indelicato PA. Bone tunnel enlargement after anterior cruciate ligament replacement. Am J Sports Med. 1994;22:410-4.

14. Clatworthy MG, Annear P, Bulow JU, Bartlett RJ. Tunnel widening in anterior cruciate ligament reconstruction: a prospective evaluation of hamstring and patella tendon grafts. Knee Surg Sports Traumatol Arthrosc. 1999;7:138-45.

15. Ma CB, Francis K, Towers J, Irrgang J, Fu FH, Harner CH. Hamstring anterior cruciate ligament reconstruction: a comparison of bioabsorbable interference screw and endobutton-post fixation. Arthroscopy. 2004;20:122-8.

16. Simonian PT, Erickson MS, Larson RV, O’Kane J W. Tunnel expansion after hamstring anterior cruciate ligament reconstruction with 1-incision EndoButton femoral fixation. Arthroscopy. 2000;16:707-14.

17. Kellgren JH, Lawrence JS. Radiological assessment of osteoarthrosis. Ann Rheum Dis. 1957;16:494-502.

18. Majewski M, Susanne H, Klaus S. Epidemiology of athletic knee injuries: a 10-year study. Knee. 2006;13:184-8.

19. Carson EW. Fact, myth, or common sense: anterior cruciate ligament reconstruction graft selection. Orthopedics. 1999;22:567-8.

20. Shelbourne KD, Nitz P. Accelerated rehabilitation after anterior cruciate ligament reconstruction. Am J Sports Med. 1990;18:292-9.

21. Ahmad CS, Gardner TR, Groh M, Arnouk J, Levine WN. Mechanical properties of soft tissue femoral fixation devices for anterior cruciate ligament reconstruction. Am J Sports Med. 2004;32:635-40.

22. Laxdal G, Kartus J, Eriksson BI, Faxen E, Sernert N, Karlsson J. Biodegradable and metallic interference screws in anterior cruciate ligament reconstruction surgery using hamstring tendon grafts: prospective randomized study of radiographic results and clinical outcome. Am J Sports Med. 2006;34:1574-80.

23. Wilson TC, Kantaras A, Atay A, Johnson DL. Tunnel enlargement after anterior cruciate ligament surgery. Am J Sports Med. 2004;32:543-9.

24. Buck DC, Simonian PT, Larson RV, Borrow J, Nathanson DA. Timeline of tibial tunnel expansion after single-incision 
hamstring anterior cruciate ligament reconstruction. Arthroscopy. 2004;20:34-6.

25. Fink C, Zapp M, Benedetto KP, Hackl W, Hoser C, Rieger M. Tibial tunnel enlargement following anterior cruciate ligament reconstruction with patellar tendon autograft. Arthroscopy. 2001;17:138-43.

26. Peyrache MD, Djian P, Christel P, Witvoet J. Tibial tunnel enlargement after anterior cruciate ligament reconstruction by autogenous bone-patellar tendon-bone graft. Knee Surg Sports Traumatol Arthrosc. 1996;4:2-8.

27. Pinczewski LA, Deehan DJ, Salmon LJ, Russell VJ, Clingeleffer A. A five-year comparison of patellar tendon versus four-strand hamstring tendon autograft for arthroscopic reconstruction of the anterior cruciate ligament. Am J Sports Med. 2002;30:523-36. 